\title{
Front Matter: Volume 6582
}

, "Front Matter: Volume 6582," Proc. SPIE 6582, Nonlinear Optics and Applications II, 658201 (18 May 2007); doi: 10.1117/12.742939

SDIE Event: International Congress on Optics and Optoelectronics, 2007, Prague, Czech Republic 


\title{
PROCEEDINGS OF SPIE
}

\section{Nonlinear Optics \\ and Applications II}

\author{
Mario Bertolotti \\ Editor \\ 16-18 April 2007 \\ Prague, Czech Republic \\ Sponsored by \\ SPIE Europe \\ Cooperating Organizations \\ SPIE Czech Republic Chapter \\ COST \\ ESF-European Science Foundation (France) \\ Czech and Slovak Society for Photonics \\ ePIXnet (Belgium) \\ Fyzikálni Ústav (Czech Republic) \\ Meta Morphose (United Kingdom) \\ NEMO_Network of Excellence in Micro-Optics (Belgium) \\ PhOREMOST \\ SPIE Poland Chapter \\ Published by \\ SPIE
}


The papers included in this volume were part of the technical conference cited on the cover and title page. Papers were selected and subject to review by the editors and conference program committee. Some conference presentations may not be available for publication. The papers published in these proceedings reflect the work and thoughts of the authors and are published herein as submitted. The publisher is not responsible for the validity of the information or for any outcomes resulting from reliance thereon.

Please use the following format to cite material from this book:

Author(s), "Title of Paper," in Nonlinear Optics and Applications II, edited by Mario Bertolotti, Proceedings of SPIE Vol. 6582 (SPIE, Bellingham, WA, 2007) Article CID Number.

ISSN 0277-786X

ISBN 9780819467102

Published by

SPIE-The International Society for Optical Engineering

P.O. Box 10, Bellingham, Washington $98227-0010$ USA

Telephone 1 360/676-3290 (Pacific Time) · Fax 1 360/647-1445

http://www.spie.org

Copyright (C) 2007, The Society of Photo-Optical Instrumentation Engineers

Copying of material in this book for internal or personal use, or for the internal or personal use of specific clients, beyond the fair use provisions granted by the U.S. Copyright Law is authorized by SPIE subject to payment of copying fees. The Transactional Reporting Service base fee for this volume is $\$ 18.00$ per article (or portion thereof), which should be paid directly to the Copyright Clearance Center (CCC), 222 Rosewood Drive, Danvers, MA 01923. Payment may also be made electronically through CCC Online at http://www.copyright.com. Other copying for republication, resale, advertising or promotion, or any form of systematic or multiple reproduction of any material in this book is prohibited except with permission in writing from the publisher. The CCC fee code is 0277$786 \times / 07 / \$ 18.00$.

Printed in the United States of America.

Publication of record for individual papers is online in the SPIE Digital Library.

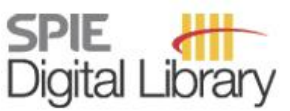

SPIEDigitalLibrary.org

Pagination: Proceedings of SPIE follow an e-First publication model, with papers published first online and then in print and on CD-ROM. Papers are published as they are submitted and meet publication criteria. A unique, consistent, permanent citation identifier (CID) number is assigned to each article at the time of the first publication. Utilization of CIDs allows articles to be fully citable as soon they are published online, and connects the same identifier to all online, print, and electronic versions of the publication. SPIE uses a six-digit CID article numbering system in which:

- The first four digits correspond to the SPIE volume number.

- The last two digits indicate publication order within the volume using a Base 36 numbering system employing both numerals and letters. These two-number sets start with 00, 01, 02, 03, 04, 05, 06, 07 , $08,09,0 \mathrm{~A}, 0 \mathrm{~B} \ldots \mathrm{.}$. Z, followed by 10-1Z, 20-2Z, etc.

The CID number appears on each page of the manuscript. The complete citation is used on the first page, and an abbreviated version on subsequent pages. 


\section{Contents}

ix Conference Committee

xi Liquid crystalline nonlinear optical metamaterials with low-loss tunable negative-zeropositive refractive indices (Plenary Paper) [6587-203]

I. C. Khoo, A. Diaz, D.-H. Kwon, D. H. Warner, Pennsylvania State Univ. (USA)

SESSION 1 SPECTROSCOPY

658208 Nonparaxial evolution of femtosecond optical pulses. [6582-05]

L. M. Kovachev, Institute of Electronics, BAS (Bulgaria)

\section{SESSION 2 ULTRAFAST PULSES AND SOLITONS}

65820A Analytical dynamics of optical similariton solutions of the amplified nonlinear Schrödinger equation with varying parameters [6582-07]

S. Wabnitz, CNRS, Univ. de Bourgogne (France)

65820B Dark solitary vortices in defocusing media [6582-08]

N. K. Efremidis, National Technical Univ. of Athens (Greece) and Univ. of Crete (Greece);

P. Papagiannis, N. Moshonas, Y. Kominis, K. Hizanidis, National Technical Univ. of Athens

(Greece); B. A. Malomed, Tel Aviv Univ. (Israel)

$65820 \mathrm{E}$ Self-induced waveguides created by second harmonic generation in lithium niobate [6582-11]

F. Pettazzi, Univ. La Sapienza e CNISM (Italy) and Univ. de Franche-Comté (France);

V. Coda, M. Chauvet, Univ. de Franche-Comté (France); E. Fazio, Univ. La Sapienza

e CNISM (Italy)

SESSION 3 PHOTONIC CRYSTALS I

$65820 \mathrm{G}$ Enhanced nonlinear interactions in optical slow wave structures and the limiting role of disorder [6582-14]

S. Mookherjea, A. Oh, Univ. of California/San Diego (USA)

\section{SESSION 4 PHOTONIC CRYSTALS II}

$65820 \mathrm{~K}$ The propagation of nonlinear electromagnetic TE surface waves in magnetic super lattices (LANS) film [6582-18]

M. M. Shabat, Max-Planck-Institut für Physik komplexer Systeme (Germany); H. M. Mousa,

Al Azhar Univ. (Palestinian Authority) 
$65820 \mathrm{~L}$ Periodically corrugated nonlinear planar waveguide as a source of squeezed light. [6582-19] J. Peřina, Jr., O. Haderka, Palacký Univ. (Czech Republic); C. Sibilia, M. Bertolotti, Univ. La Sapienza di Roma (Italy); M. Scalora, Redstone Arsenal (USA)

65820M AIGaAs waveguide generation of counterpropagating twin photons [6582-20]

L. Lanco, S. Ducci, J. Likforman, M. Ravaro, V. Berger, G. Leo, Univ. Paris-Diderot (France)

\section{SESSION 5 MATERIALS AND APPLICATIONS}

65820N Nonlinear spectroscopy and applications: time-resolved nonlinear spectroscopy of silicon nanocrystals (Invited Paper) [6582-21]

P. Malý, N. Neudert, K. Židek, M. Bittner, F. Trojánek, Charles Univ. in Prague (Czech Republic)

$65820 \mathrm{P}$ Second harmonic generation from bulk glassceramics containing laser-poled dielectric nanocrystals [6582-23]

A. Paleari, Univ. degli Studi di Milano-Bicocca (Italy); S. Brovelli, Univ. College London (United Kingdom); R. Lorenzi, N. Chiodini, G. Spinolo, Univ. degli Studi di Milano-Bicocca (Italy)

65820S Flat-top temperature tuning response in periodically poled nonlinear crystals [6582-27] H. E. Major, A. C. Peacock, C. Farrell, Univ. of Southampton (United Kingdom);

C. B. E. Gawith, P. G. R. Smith, Univ. of Southampton (United Kingdom) and Stratophase Ltd. (United Kingdom); M. Rusu, M. Guina, O. G. Okhotnikov, Tampere Univ. of Technology (Finland)

\section{SESSION 6 PARAMETRIC AND RAMAN LASERS}

65820T Ring picosecond optical parametric oscillator pumped synchronously, intracavity, by a mode-locked Nd:YVO 4 laser [6582-28]

A. Zavadilová, V. Kubeček, Czech Technical Univ. (Czech Republic); J.-C. Diels, CHTM, Univ. of New Mexico (USA)

$65820 \mathrm{U}$ Wavelength-agile coherent tunable mid-IR ZGP-OPO source and its applications [6582-29] K. Miyamoto, Tohoku Univ. (Japan) and RIKEN Sendai (Japan); K. M. Abedin, TohokU Univ. (Japan); H. Ito, Tohoku Univ. (Japan) and RIKEN Sendai (Japan)

$65820 \mathrm{~W}$ Mitigating heat dissipation in a hydrogen-based Raman laser using coherent anti-Stokes Raman scattering [6582-31]

N. Vermeulen, C. Debaes, H. Thienpont, Vrije Univ. Brussel (Belgium)

65820X Enhancement methods for CARS-based heat mitigation and application to near- and midinfrared silicon-based Raman lasers [6582-32]

N. Vermeulen, C. Debaes, H. Thienpont, Vrije Univ. Brussel (Belgium) 
65820Y Narrowing of oscillation linewidth in a quasi-monolithic THz-wave parametric oscillator [6582-33]

H. Minamide, R. Guo, T. Ikari, RIKEN Sendai (Japan); H. Ito, RIKEN Sendai (Japan) and RIEC

Tohoku Univ. (Japan)

$65820 Z$ Detection of coherent tunable THz-wave using of stimulated polariton scattering in MgO:LiNbO3 [6582-34]

R. GUo, T. Ikari, H. Minamide, RIKEN Sendai (Japan); H. Ito, RIKEN Sendai (Japan) and RIEC

Tohoku Univ. (Japan)

658211 Improved emission and coherent detection of few-cycle terahertz transients using laser pulses at $1.5 \mu \mathrm{m}[6582-36]$

A. Schneider, M. Stillhart, Z. Yang, F. Brunner, P. Günter, ETH Zürich (Switzerland)

\section{SESSION 8 APPLICATIONS}

658212 Perturbation theory of dispersion-managed fiber solitons [6582-37]

M. F. S. Ferreira, M. H. Sousa, Univ. of Aveiro (Portugal)

658213 Stimulated Brillouin scattering in graded index multimode optical fiber by excitation of the fundamental mode only [6582-38]

P. Lenke, N. Nöther, Federal Institute for Materials Research and Testing (Germany)

658214 Novel optical CDMA plafform for avionics applications [6582-39]

I. Glesk, Comenius Univ. (Slovak Republic) and Princeton Univ. (USA); P. R. Prucnal, Princeton Univ. (USA)

658215 Nonlinear light propagation in photonic crystal fibers filled with nematic liquid crystals [6582-40]

K. A. Rutkowska, U. A. Laudyn, R. T. Rutkowski, M. A. Karpierz, T. R. Woliński, Warsaw Univ. of Technology (Poland); J. Wójcik, Maria Skłodowska Curie Univ. (Poland)

658216 Nonlinear frequency conversion in double core photonic crystal fibers [6582-41] I. Bugar, D. Lorenc, International Laser Ctr. (Slovak Republic); I. V. Fedotov, A. B. Fedotov, M.V. Lomonosov Moscow State Univ. (Russia); R. Buczynski, Warsaw Univ. (Poland); D. Pysz, Institute of Electronics Materials Technology (Poland); F. Uherek, International Laser Ctr. (Slovak Republic); A. M. Zheltikov, M.V. Lomonosov Moscow State Univ. (Russia)

\section{POSTER SESSION}

658218 Dark soliton dynamics and interactions in dynamically photo-induced lattices: interaction with a continuous wave [6582-42]

I. Tsopelas, Y. Kominis, P. Papagiannis, N. Moshonas, L. Halastanis, N. Efremidis, K. Hizanidis, National Technical Univ. of Athens (Greece)

65821D Measurement of thin films using slightly dispersive spectral interferometry [6582-48] R. Chlebus, P. Hlubina, D. Ciprian, J. Luňáček, Technical Univ. Ostrava (Czech Republic) 
65821E Dynamics of solitary waves in nonlinear media with Bragg gratings [6582-49]

P. Papagiannis, L. Halastanis, I. Tsopelas, N. Moshonas, Y. Kominis, K. Hizanidis, National Technical Univ. of Athens (Greece)

65821 F Investigation of spatiotemporal optical beam reallocation [6582-50]

N. Moshonas, Y. Kominis, National Technical Univ. of Athens (Greece); N. Efremidis, National Technical Univ. of Athens (Greece) and Univ. of Crete (Greece); M. Manousakis,

K. Hizanidis, National Technical Univ. of Athens (Greece)

$65821 \mathrm{G}$ Real-time gas density measurement using a ring cavity terahertz parametric oscillator [6582-51]

S. Ohno, R. Guo, H. Minamide, RIKEN Sendai (Japan); H. Ito, RIKEN Sendai (Japan) and RIEC Tohoku Univ. (Japan)

$65821 \mathrm{H} \quad$ Ultrafast spectroscopy of semiconductor Bragg reflectors [6582-52]

M. V. Ermolenko, S. A. Tikhomirov, V. V. Stankevich, O. V. Buganov, S. V. Gaponenko, Institute of Molecular and Atomic Physics (Belarus); P. I. Kuznetsov, G. G. Yakuscheva, Institute of Radioengineering and Electronics (Russia)

658211 Femtosecond absorption bleaching in glasses with semiconductor nanoparticles [6582-53] A. Lipovskii, St.-Petersburg State Polytechnic Univ. (Russia); M. Halonen, University of Joensuu (Finland); E. V. Kolobkova, St.-Petersburg State Technological Institute (Russia); Y.P. Svirko, University of JoensuU (Finland)

$65821 \mathrm{~K}$ Semiconductor-polymer composite with strong nonlinear optical properties [6582-55] J. Franc, Charles Univ. (Czech Republic); V. Babentsov, Institute of Semiconductor Physics (Ukraine); P. Feychuk, Chernivtsi State Univ. (Ukraine); J. Kunc, P. Hlídek, Charles Univ. (Czech Republic)

$65821 \mathrm{M}$ All-optical ultrafast packet contention detection based on SOAs for $2 \times 2$ photonic nodes [6582-57]

M. Scaffardi, F. Fresi, G. Berrettini, G. Meloni, N. Andriolli, P. Castoldi, Scuola Superiore Sant'Anna (Italy); L. Poti, A. Bogoni, Consorzio Nazionale Interuniv. per le Telecomunicazioni (Italy)

$65821 \mathrm{~N}$ Four-wave mixing in intersubband transitions of a semiconductor quantum well structure [6582-58]

E. Paspalakis, A. Kanaki, A. F. Terzis, Univ. of Patras (Greece)

65821P Dark photovoltaic spatial solitons: experiment and numerical solution [6582-60]

M. Bodnár, Czech Technical Univ. in Prague (Czech Republic)

658215 Tunable optical filter for wavelength division multiplexing using photorefractive planar waveguide [6582-63]

S. Honma, H. Ito, M. Komatsu, S. Muto, Univ. of Yamanashi (Japan); A. Okamoto, Hokkaido Univ. (Japan) 
$65821 \mathrm{U}$ Efficient coupling for bidirectional pumping in EDFA with two mutually-pumped phaseconjugate mirrors [6582-65]

S. Kano, A. Okamoto, Hokkaido Univ. (Japan); Y. Takayama, National Institute of Information and Communications Technology (Japan); K. Sato, Hokkai-Gakuen Univ. (Japan)

Author Index 
Downloaded From: https://www.spiedigitallibrary.org/conference-proceedings-of-spie on 26 Apr 2023

Terms of Use: https://www.spiedigitallibrary.org/terms-of-use 


\title{
Conference Committee
}

\author{
Symposium Chairs
}

Pavel Tománek, Brno University of Technology (Czech Republic) Miroslav Hrabovský, Palacký University (Czech Republic)

Hugo Thienpont, Vrije Universiteit (Belgium)

Symposium Honorary Chair

Karel Jungwirth, Institute of Physics (Czech Republic)

Conference Chair

Mario Bertolotti, Università degli Studi di Roma, La Sapienza (Italy)

Cochairs

Joseph W. Haus, University of Dayton (USA)

Alexei M. Zheltikov, M.V. Lomonosov Moscow State University (Russia)

Program Committee

Werner J. Blau, The University of Dublin, Trinity College (Ireland)

Bruno Crosignani, Università dell'Aquila (Italy)

Claude Fabre, Université Pierre et Marie Curie (France)

Hiromasa Ito, RIKEN (Japan) and Tohoku University (Japan)

Jan Perina, Univerzita Palackeho v Olomouci (Czech Republic)

David C. Smith, University of Southampton (United Kingdom)

Dietrich von der Linde, Universität Duisburg-Essen (Germany)

\section{Session Chairs}

1 Spectroscopy

Mark A. Foster, Cornell University (USA)

2 Ultrafast Pulses and Solitons

Hiromasa Ito, RIKEN (Japan) and Tohoku University (Japan)

Takayoshi Kobayashi, The University of Tokyo (Japan)

3 Photonic Crystals I

Concita Sibilia, Università degli Studi di Roma, La Sapienza (Italy)

4 Photonic Crystals II

Aharon J. Agranat, The Hebrew University of Jerusalem (Israel) 
$5 \quad$ Materials and Applications

Stefan Wabnitz, Université de Bourgogne (France)

$6 \quad$ Parametric and Raman Lasers

Hiromasa Ito, RIKEN (Japan) and Tohoku University (Japan)

$7 \quad$ Terahertz

Willie J. Padilla, Boston College (USA)

8 Applications

David C. Smith, University of Southampton (United Kingdom) 


\title{
Liquid Crystalline Nonlinear Optical Metamaterials with Low-Loss Tunable Negative-Zero-Positive Refractive Indices
}

\author{
I. C. Khoo, A. Diaz, D. -H. Kwon and D. H Werner \\ Electrical Engineering Department, Pennsylvania State University, University Park, PA 16802
}

\begin{abstract}
We describe a class of liquid crystalline photonic metamaterials that exhibit tunable negative-zero-positive refractive indices. As a result of the extreme sensitivity of the nematic liquid crystal constituent, these metamaterials also exhibit extraordinarily large optical nonlinearities associated with the optical field induced director axis reorientation and birefringence change. Incorporation of a gain medium such as laser dye reduces losses of the metamaterial.
\end{abstract}

Keywords: Liquid crystals, nano-spheres, supra optical nonlinearity, negative and zero index, tunable metamaterials

\section{INTRODUCTION}

Current material systems that exhibit negative index properties tend to be passive and highly lossy. Recently, we demonstrated two types of nano-dispersed liquid crystalline metamaterials [1,2] whose effective refractive index can be tuned from negative through zero to positive values over a very wide spectral range. The tunability is provided by incorporating electro-optics active and nonlinear-optical nematic liquid crystals [3]. In this paper, we also explore the ultimate optical nonlinearity possible in such metamaterials due to the supra-nonlinear optical properties of the nematic liquid crystal constituents. Furthermore, we demonstrate that one could reduce the loss associated with the dielectric resonances by introducing laser dye in the system.

\section{NANO-SPHERES DISPERSED LIQUID CRYSTALLINE METAMATERIALS}

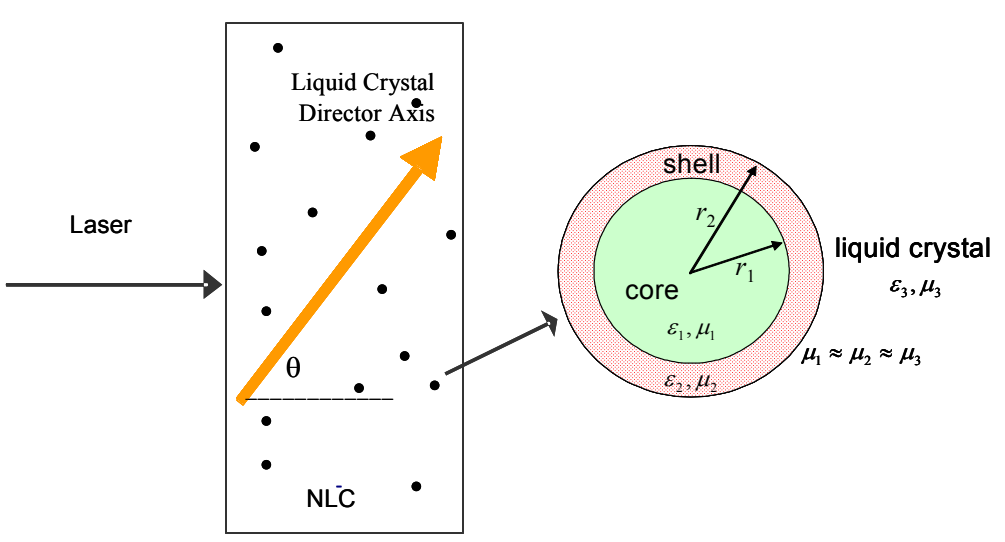

Fig 1. Schematic depiction of nematic liquid crystal containing nanospheres and a 'blow-up' view of the core-shell nano-spheres.
Fig 1 shows a schematic of an aligned nematic liquid crystal doped with randomly dispersed core-shell nano-spheres. The nematic liquid crystal can be in pure or doped (e.g. by Methyl-Red) forms, while the nano-spheres can be in the form of simple single constituent metallic (gold, silver, semiconductor) spheres or core-shell structures. The electromagnetic analysis for such metamaterials is considerably simpler than other schemes involving metallic spheres dispersed in liquid crystal waveguides as we can employ the effective medium approach.

We have studied a variety of nanospheres in various combinations and liquid crystal alignments. Here we summarize the principal results for the case of core-shell polaritonic and Drude-type nano-spheres discussed in the literature $[1,4]$. All constituent materials are non-magnetic with relative permeability equal to 1 . For a polaritonic core, its permittivity is given by: 


$$
\varepsilon_{1}=\varepsilon(\infty)\left(1+\frac{\omega_{L}^{2}-\omega_{T}^{2}}{\omega_{T}^{2}-\omega^{2}-i \omega \gamma_{1}}\right)
$$

where $\varepsilon(\infty)$ is the high-frequency limit of the permittivity, $\omega$ is the incident frequency, $\omega_{T}$ is the transverse optical phonon frequency, $\omega_{L}$ is the longitudinal optical phonon frequency, and $\gamma_{1}$ is the damping coefficient.

The shell can be a polaritonic or a Drude material with a permittivity of the form:

$$
\varepsilon_{2}=1-\frac{\omega_{p}^{2}}{\omega^{2}+i \omega \gamma_{2}},
$$

where $\omega_{p}$ is the plasma frequency and $\gamma_{2}$ is the damping term. The optical permittivity of the host nematic liquid crystal (NLC) for a linearly polarized light incident at an oblique angle $\theta$ is given by [3]

$$
\varepsilon_{3}=\frac{\varepsilon_{e} \varepsilon_{o}}{\varepsilon_{e} \cos ^{2} \theta+\varepsilon_{o} \sin ^{2} \theta}
$$

where $\varepsilon_{e}$ and $\varepsilon_{o}$ are the respective permittivities for light polarized parallel and perpendicular to the director axis $\hat{n}$, and $\theta$ is the angle made by the director axis with the optical wave vector $k_{0}$. Note that $\varepsilon_{3}$ does not carry any resonant dependence except for some small variation over the optical- infrared wavelength regime of interest here.

For a fixed incident angle, the director axis orientation $\theta$ with respect to the optical wave vector can be modulated either electrically [by an ac bias field] or optically [through the optical intensity dependent director axis reorientation effect (3)]. In both cases, the maximum reorientation angle is $\pi / 2$, corresponding to an extraordinary refractive index change from $n_{\mathrm{o}} \sim 1.4[\theta=0]$ to $n_{\mathrm{e}} \sim 2[\theta=\pi / 2]$ i.e. permittivity change from $\varepsilon_{\mathrm{o}}=2$ to $\varepsilon_{\mathrm{e}}=4$ ( $\varepsilon_{\mathrm{e}}$ for extraordinary and $\varepsilon_{\mathrm{o}}$ for ordinary waves). Such changes in the host index give rise to large changes in the effective refractive index of the nano-dispersed liquid crystal (NDLC) that in many cases. We have previously shown that the effective refractive indices of these metamaterials could be unusual negative-zero regions in some cases.

The effective refractive index of the nano-dispersed NLC is calculated by using the Maxwell Garnet mixing rule [5]. We begin by calculating the effective permittivity and permeability $\varepsilon_{r}^{e f f}$ and $\mu_{r}^{\text {eff }}$ for NDLC as follow.

$$
\begin{gathered}
\varepsilon_{r}^{e f f}=\varepsilon_{3}\left(\frac{k_{3}^{3}+j 4 \pi N a_{1}}{k_{3}^{3}-j 2 \pi N a_{1}}\right), \\
\mu_{r}^{e f f}=\frac{k_{3}^{3}+j 4 \pi N b_{1}}{k_{3}^{3}-j 2 \pi N b_{1}} .
\end{gathered}
$$

where

$$
k_{3}=\sqrt{\varepsilon_{3}} k_{0}=\left(\frac{\varepsilon_{e} \varepsilon_{o}}{\varepsilon_{e} \cos ^{2} \theta+\varepsilon_{o} \sin ^{2} \theta}\right)^{1 / 2} k_{0}
$$

In equations (4a-4b), $a_{1}$ and $b_{1}$ are the MIE scattering coefficients of the coated dielectric sphere [5], $N$ is the volume density of the spheres $\left(N=3 f / 4 \pi r_{2}^{3}\right.$ ) and $f$ is the filling fraction of the composite. Note that the Maxwell Garnett mixing method is valid only for small filling fractions, i.e. $f<<1$.

Some exemplary results for the effective refractive indices are shown in Fig. 2 calculated with the set of parameters for the constituent core, shell and host medium $[1,4]: \varepsilon(\infty)=17, \omega_{L} / 2 \pi=570 \mathrm{THz}, \omega_{T} / 2 \pi=240 \mathrm{THz}, \gamma_{1} / 2 \pi=2.5$ $\mathrm{THz}, \mu_{1}=\mu_{2}=\mu_{3}=1, \gamma_{2}=\omega_{p} / 60, \omega_{p} / 2 \pi=134 \mathrm{THz}, r_{1}=0.13 \mu \mathrm{m}, r_{2}=0.143 \mu \mathrm{m}$ and a filling fraction $f=0.1$. In general, as a result of incorporating nano-spheres with higher dielectric constants than the host nematic liquid crystals (NLC), the effective birefringence $n_{\mathrm{e}}-n_{\mathrm{o}}$ of the metamaterial is larger than the birefringence of the host liquid crystal ( $n_{\mathrm{e}}$ 
$\left.-n_{\mathrm{o}}\right)_{\mathrm{NLC}}$, which is assumed to be 0.59 in these calculations [corresponding to the NLC dielectric constants varying from 2 to 4]. In the low-loss frequency regime (around $50-70 \mathrm{THz}$ ) where the imaginary component of $n_{\text {eff }}$ is small, the effective [real part] refractive index change $\Delta \mathrm{n}$ is $\sim 0.75$ [at $50 \mathrm{THz}$ ] or 0.9 [at $70 \mathrm{THz}$ ] as the NLC permittivity is varied from 2 to 4 . At $80 \mathrm{THz}$, where the loss is still acceptably low, the effective index change is even larger [ 1.1].

It is interesting to note that for some frequency interval around $106 \mathrm{THz}$, the effective refractive index of the liquid crystal metamaterial can be negative and is tunable from negative, through zero, to positive values as the host NLC dielectric constant (birefringence) is varied. For other parameter sets and filling fractions, the operating frequencies for these negative-zero-positive tunable refractive indices could be anywhere from the visible, near-, mid and far-IR region through $\mathrm{THz}$ and into the microwave regime, mainly due to the extremely broadband and large birefringence of the nematic liquid crystal host.
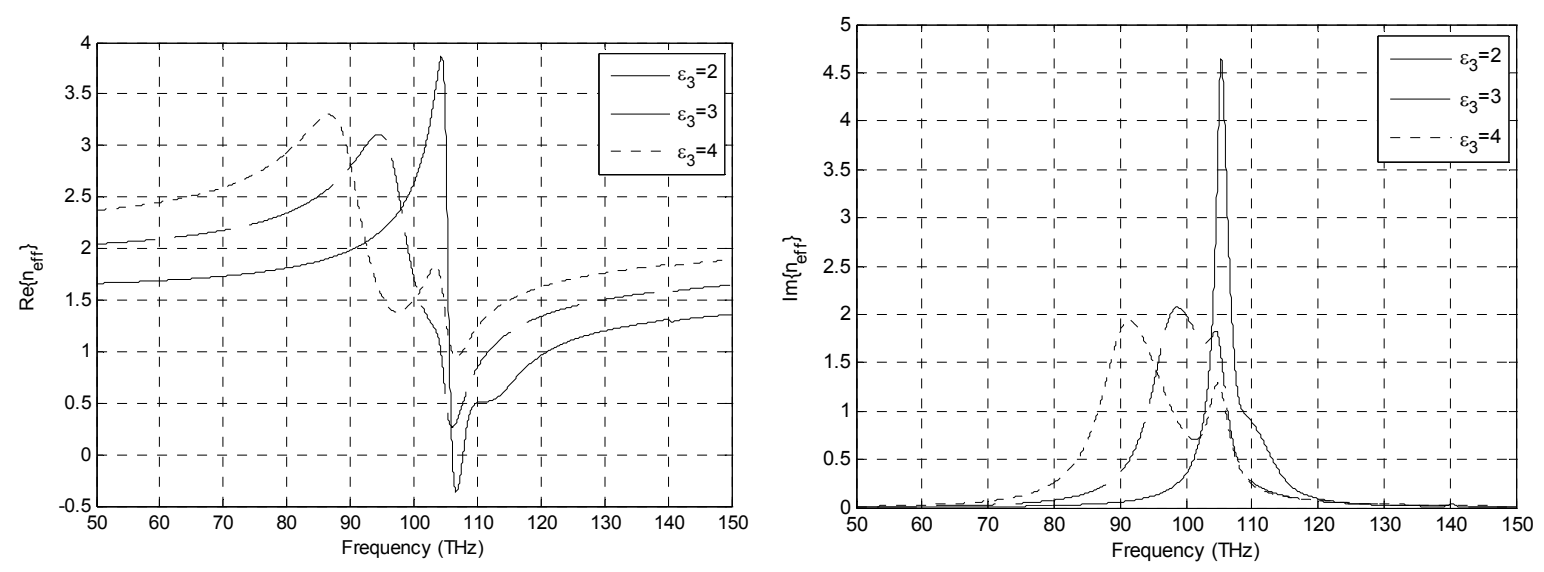

Fig. 2 Real [left] and Imaginary [right] parts of the complex effective refractive index of the core-shell nano-spheres dispersed nematic liquid crystals showing tunable refractive index from negative to positive values.

\section{LOSS REDUCTION WITH GAIN (DYE-DOPED LIQUID CRYSTAL HOST)}

In spite of these unusual and remarkable properties of the liquid crystalline metamaterials, there is the inevitable presence of high optical losses in the same frequency region. The inevitability is associated with the relationship [Kramers-Kronig] between the real and imaginary part of the susceptibility of the medium. Nevertheless, within the constraint of the Kramers-Kronig relationship, one could ascertain an operation parameter set in which the loss is minimal, while the real part of the index is in the desirable range. We have investigated the possibility of reducing the loss by introducing laser dyes in the host liquid crystal matrix to provide gain [6]. Although the actual molecular levels and transitions in dye molecules are much more complex, we illustrate the effects by a two-level inverted system characterized by a susceptibility of the form $\chi_{g}=\chi_{g}^{\prime}+i \chi_{g}^{\prime \prime}$. Accordingly, the dielectric constant of the host [liquid crystal] becomes $\varepsilon_{h}=\varepsilon_{3}+\chi_{g}$. Following Yariv [7], the imaginary part of the susceptibility $\chi_{\mathrm{g}}$ is given by (with an appropriate change of sign because of different conventions used here):

$$
\chi_{g}^{\prime \prime}=\frac{\left(N_{1}-N_{2}\right) \lambda^{3}}{8 \pi^{3} n_{3} t_{\text {spont }} \Delta v} \frac{1}{1+4\left(v-v_{0}\right)^{2} /(\Delta v)^{2}}=A \times \frac{1}{v^{3} n_{3} \Delta v} \times \frac{1}{1+4\left(v-v_{0}\right)^{2} /(\Delta v)^{2}}
$$

with $A=\frac{\left(N_{1}-N_{2}\right) c^{3}}{8 \pi^{3} t_{\text {spont }}}=\chi_{\max }^{\prime \prime}\left(v=v_{0}\right) \times v_{0}^{3} n_{3} \Delta v$ where $\chi_{\max }^{\prime \prime}\left(v=v_{0}\right)$ is the imaginary part of the susceptibility at the resonant frequency $\chi_{\max }^{\prime \prime}\left(v=v_{0}\right)$ and $\Delta v$ is the transition linewidth, and $n_{3}$ is the refractive index of the surrounding medium. From $\chi_{g}^{\prime \prime}$, the real part of the susceptibility of the gain medium may be derived from the Kramers-Kronig relations (7): 


$$
\chi_{g}^{\prime}=\frac{\left(N_{1}-N_{2}\right) \lambda^{3}}{8 \pi^{3} n_{3} t_{\text {spoon }} \Delta v} \frac{2\left(v-v_{0}\right) / \Delta v}{1+4\left(v-v_{0}\right)^{2} /(\Delta v)^{2}}=\chi_{g}^{\prime \prime} \cdot \frac{2\left(v-v_{0}\right)}{\Delta v}
$$
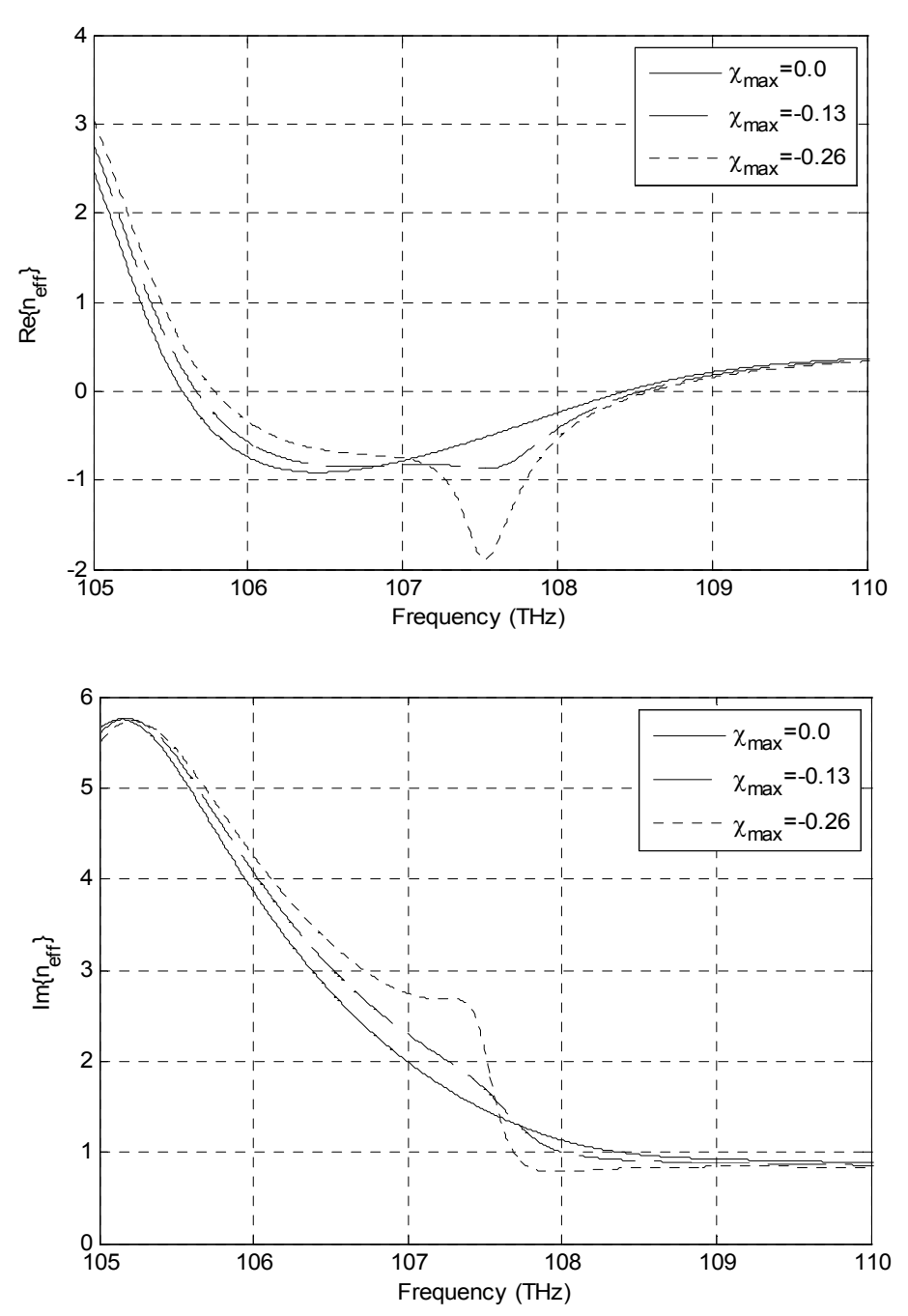

Fig. 3. Real [upper] and imaginary [lower] parts of the refractive index of nano-dispersed dye-doped nematic liquid crystals showng enhanced negative index behavior and a lowered loss [smaller imaginary refractive index]
Fig. 3 show the real and imaginary part of the effective refractive index of the nano- and dyedoped nematic liquid crystals for three representative values of the imaginary part of the susceptibility at the resonant frequency $\chi_{\max }[0$; 0.13 and 0.26 ] and a similar set of core-shell annospheres material parameters. It is clear that one could maintain negative-zero [real] refractive index while reducing the $\operatorname{loss}\left(\operatorname{Im}\left[n_{\text {eff }}\right]\right)$ considerably. It is interesting to note that the real part of the refractive index $\left(\operatorname{Re}\left[n_{\text {eff }}\right]\right)$ could also be decreased even further with the incorporation of the gain medium (for $\chi_{\max }=-0.13$ ).

\section{Liquid crystal clad metallo-dielectric nano-structures for tunable negative-zero- positive refractive indices.}

Another nematic liquid crystal based metamaterial [2] capable of tunable negative-zero-positive refractive indices we have studied is schematically depicted in fig. 4. It consists of two aligned nematic liquid crystal layers sandwiching a metallo-dielectric nanostructure. The latter comprises a magnetic resonator made of two strips of silver of thickness $30 \mathrm{~nm}$ separated by a thin layer of alumina of thickness $20 \mathrm{~nm}$. Negative permittivity needed for negative-index behavior is provided by thin silver films bounding the periodic array of magnetic resonators. The space between neighboring magnetic resonators is filled with silica. The optical properties of the metamaterial are analyzed using a rigorous full-wave electromagnetic scattering analysis based on the finite-element boundary-integral method [8]. Electric field values in a single unit cell of an infinitely periodic structure are determined by imposing periodic boundary conditions in the computational domain. Once the complex reflection and transmission coefficients are determined from the numerical analysis, the effective complex index of refraction can be unambiguously determined from well-established inversion procedures $[9,10]$.

The plots of the complex refractive index $n=n^{\prime}+n$ " in Fig. 4 for two different incident light wavelengths as a function of the liquid crystal dielectric constant $\varepsilon_{\mathrm{LC}}$ show dramatic changes in the effective refractive index created by the inclusion of these 'resonant' structures. For example, the lower right solid curve for incident light wavelength $\lambda=$ $1.45 \mu \mathrm{m}$ shows that the effective index of the metamaterials changes by 1.3 [from -1 to 0.3 ] as the LC dielectric constant is tuned from 2.25 to 2.9 [LC index change of 0.2 from 1.5 to 1.7]. From the preceding discussion on the effect of gain, clearly if the liquid crystal is doped with a dye to provide gain, both the real and the imaginary parts of the refractive index could be further modified. 


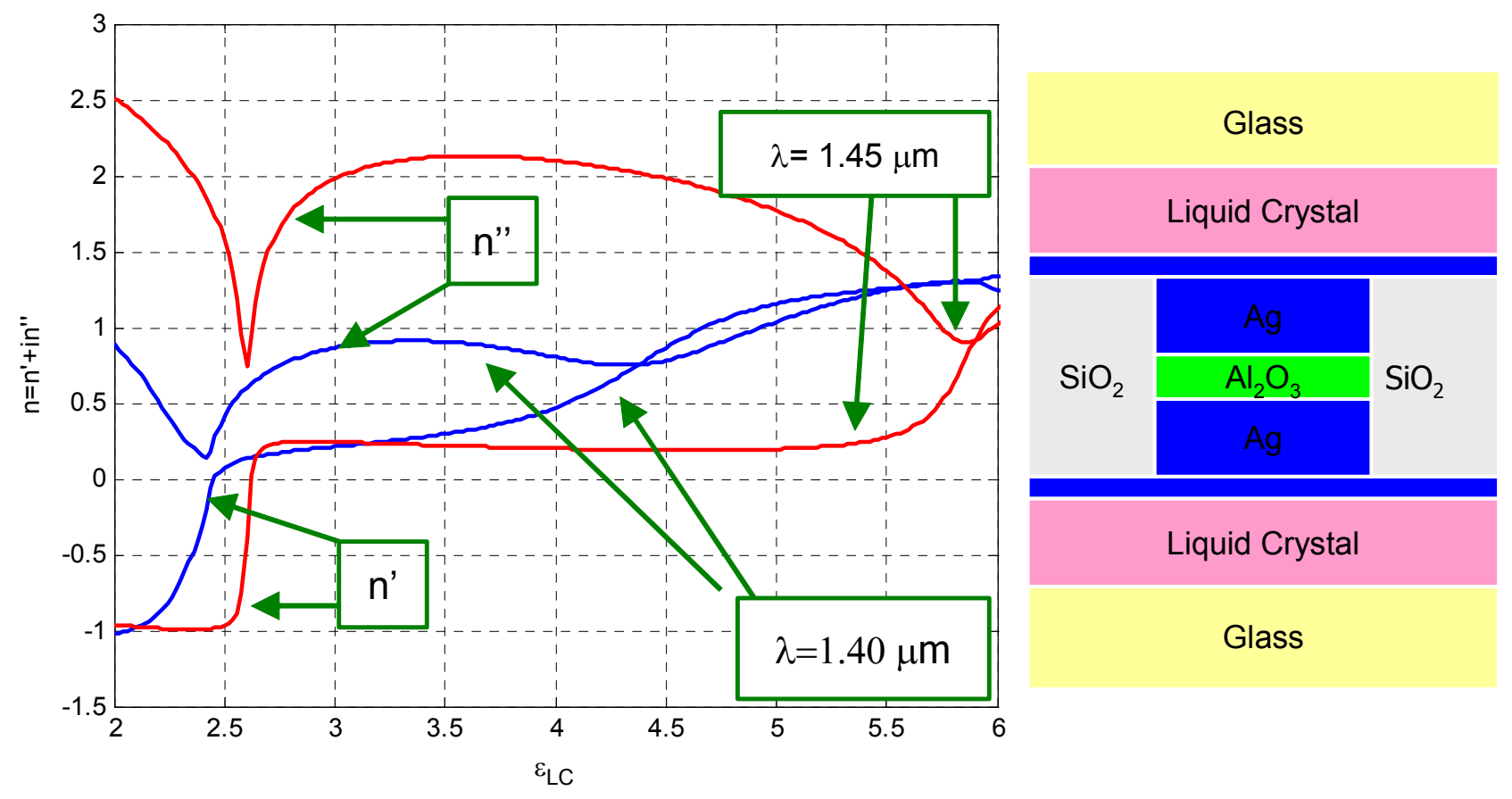

Fig. 4. Plots of real [n' - lower two curves] and imaginary [n" - upper two curves] parts of the complex refractive index of the liquid crystal clad metallo-dielectric nano-structures for various values of the liquid crystal dielectric constant and two laser wavelengths.

\section{SUPRA-OPTICAL NONLINEARITY OF NANO-DISPERSED NEMATIC LIQUID CRYSTALLINE METAMATERIALS}

As remarked earlier, the refractive index [dielectric constant] of the host liquid crystal can be electrically or optically modified. Since electrical contacts are extremely cumbersome to incorporate in the nanostructure, optical tuning is a much more desirable means. We close this paper with a discussion of the possibility of achieving high supra-optical nonlinearity in these dye-doped liquid crystal metamaterials. Nonlinear light scatterings in liquid crystals have been extensively investigated over the past two decades [3, 11-36]. Perhaps the most nonlinear mechanism is the optically induced director axis reorientation, which is characterized by an optical index change coefficient $n_{2}$ [defined by $n_{2}=\Delta n / I$, where $\Delta n$ is the light induced index change and $I$ the optical intensity]. In dye-doped nematic liquid crystals, supraoptical nonlinearities with $n_{2} \gg 1 \mathrm{~cm}^{2} / \mathrm{W}$ were first discovered [23] in methyl red dye molecules-, and subsequently also in C60- and carbon nanotube- doped NLC [26-28]. In this section, we re-examine the fundamental principles for such supra-nonlinearity and illustrate the possibility of realizing even larger optical nonlinearity with the higher-birefringence liquid crystal metamaterials discussed in the preceding sections.

Consider the basic light-LC interaction as depicted in Fig. 1. The energy density involved in reorienting the LC axis by an angle $\theta$ is $U\left[\frac{\mathrm{erg}}{\mathrm{cm}^{2}}\right] \approx K\left(\frac{\partial \theta}{\partial x}\right)^{2} L$, where $L$ is the interaction length and $K$ the LC elastic constant [3]. In a wave mixing type of interaction in which the impinging optical intensity is sinusoidal, the reorientation angle $\theta$ is of the form $\theta=\theta_{0} \sin (q x)$ where $q=2 \pi / \Lambda$ is the wave vector of the grating, and $\Lambda$ is the grating constant. In this case, we have $U_{\mathrm{LC}} \sim K \pi^{2} \theta^{2} L / \Lambda^{2}$. On the other hand, the energy provided by the light beam is $E_{\text {light }}=I \tau\left(1-\mathrm{e}^{-\alpha L}\right) \sim \alpha L I \tau$ where $\alpha$ is the loss coefficient (due to the transfer of energy from light to nematic reorientation per unit length) and $\tau$ is the response time for the process. Equating $U$ and $E_{\text {light }}$ by assuming complete conversion of transferred light energy to reorientation, 
we get $K \pi^{2} \theta^{2} / \alpha \Lambda^{2} \approx I \tau$. In the case when the liquid crystal is initially homeotropically aligned, a reorientation of angle $\theta$ will give rise to a change in the index experienced by the incident extraordinary-wave $\Delta n \sim\left(n_{\mathrm{e}}-n_{\mathrm{o}}\right) \theta^{2} \sim\left(n_{\mathrm{e}}-n_{\mathrm{o}}\right) I \tau \alpha \Lambda^{2} / K$. Writing $\Delta n=n_{2} I$ yields the nonlinear index coefficient $\mathrm{n}_{2}$

$$
n_{2} \sim\left(n_{\mathrm{e}}-n_{\mathrm{o}}\right) \tau \alpha \Lambda^{2} / K \pi^{2} .
$$

Depending on various parameters such as the birefringence and viscosity, sample thickness, and other factors like laser intensity, presence of other applied fields or photosensitive dopants, as well as the actual process involved, the value of $\mathrm{n}_{2}$ and the response time can vary considerably. In wave mixing studies, typical $\tau$ is on the order of 10's of millisecond $\left(10^{-2} \mathrm{sec}\right)$, for $\Lambda \sim 20 \mu \mathrm{m}$. Using $K \sim 10^{-7} \mathrm{erg} / \mathrm{cm},\left(n_{\mathrm{e}}-n_{\mathrm{o}}\right) \sim 0.2, \alpha \sim 100 \mathrm{~cm}^{-1}$, we have $n_{2} \sim 1 \mathrm{~cm}^{2} / \mathrm{W}$. Even larger $n_{2}$ values approaching $1000 \mathrm{~cm}^{2} / \mathrm{W}$ can be expected and have indeed been observed in dyed doped nematic liquid crystals $[23,28]$. From the expression for $n_{2}$, it is also clear that a larger effective birefringence provided by the liquid crystalline metamaterials discussed in the preceding sections would result in an enhancement of these supra-optical nonlinearities. Accordingly, the optical power density required to effect the desired refractive index change in these metamaterials could be as low as $100 \mathrm{nW}$ (nanowatt) as reported before in dye-doped nematic liquid crystals [35].

\section{CONCLUSION}

In conclusion, we have described two new forms of liquid crystalline metamaterials that possess tunable and highly nonlinear optical properties. By field induced reorientation of the liquid crystal host, thereby changing its permittivity, the material will exhibit effective refractive indices ranging from negative, through zero to positive values. The large effective refractive index change also results in enhancing the optical nonlinearity. The proposed structures are highly scalable in that the physical dimensions of its constituents can be varied over a very wide range, resulting in matamaterials whose operating wavelengths can cover the optical, through near- and far-IR to the microwave regimes. In this rather wide spectral band, nematic liquid crystals possess large birefringence and extreme photosensitivitythat would allow tuning with low applied field thresholds [3].

\section{ACKNOWLEDEGEMENT}

This work was supported by the Army Research Office, the Air Force Office of Scientific Research and Penn State MRSEC under NSF grant DMR-00800190.

\section{REFERENCES}

1. I. C. Khoo, D. H. Werner, X. Liang, A. Diaz and B. Weiner, "Nano-sphere dispersed liquid crystals for tunable negative-zero-positive index of refraction in the optical and Terahertz regimes," Optics Letts. 31, 2592 (2006).

2. D. H. Werner, D-H Kwon, I. C. Khoo, A. K. Kildeshev and V. M. Shalaev, "Liquid crystal-clad near-infrared metamaterials with tunable negative-zero-positive refractive indices," Optics Express Vol. 15, No. 6, pp 33423347, 2007.

3. $\quad$ I. C. Khoo, "Liquid Crystals" 2nd ed. (Wiley InterScience, Hoboken, NJ 2007).

4. M. Schall, H. Helm and S. R. Keiding, Int. J. Infrared Millimeter Waves 20, 595 (1999); M. S. Wheeler, J. S. Aitchison and M. Mojahedi, Phys. Rev. B 73, 045105 (2006)

5. C. F. Bohren, and D. R. Huffman, Absorption and Scattering of Light by Small Particles, (Wiley-VCH, 2004).

6. See for example, Y. Huang, Y. Zhou, and S.-T. Wu, "Spatially tunable laser emission in dye-doped photonic liquid crystals," Applied Physics Letters, vol. 88, pp. 11107-1, 2006.

7. A. Yariv, "Optical electronics in Modern Communications," [Oxford University Press, NY 19970]

8. J. L. Volakis, A. Chatterjee, and L. C. Kempel, Finite Element Method for Electromagnetics (IEEE Press, Piscataway, NJ, 1998).

9. $\quad$ D. R. Smith, S. Schultz, P. Marko`s, and C. M. Soukoulis, Phys. Rev. B 65, 195104 (2002).

10. A. V. Kildishev,W. Cai, U. K. Chettiar, H.-K. Yuan, A. K. Sarychev, V. P. Drachev, and V. M. Shalaev, J. Opt. Soc. Am. B 23(3), 423-433 (2006). 
11. I. C. Khoo and S. L. Zhuang, "Wave front conjugation in nematic liquid crystal films. IEEE J. Quant. Electronics," QE-18, p. 246, (1981); . I. C. Khoo, H. Li and Y. Liang, "Self-starting optical phase conjugation in dyed nematic liquid crystals with a stimulated thermal-scattering effect," Opt. Letts. $\underline{18}, 1490$ (1993); I. C. Khoo and Y. Liang, "Stimulated Orientational and Thermal Scatterings and Self-Starting Optical Phase Conjugation with Nematic Liquid Crystals," Phys. Rev. E62, pp 6722 -6733 (2000).

12. I. C. Khoo, "Optical-thermal induced total internal reflection to transmission switching at a glass-liquid crystal interface," Appl. Phys. Letts., 40, 645 (1982).

13. I. C. Khoo, J. Y. Hou, R. Normandin and V.C.Y. So, "Theory and experiment on optical bistability in a FabryPerot interferometer with an intracavity nematic liquid-crystal film,” Phys. Rev., A27, 3251 (1983).

14. I. C. Khoo and S. Shepard, "Submillisecond grating diffractions in nematic liquid crystal films. J. of Appl. Phys.," 54, 5491 (1983).

15. I. C. Khoo, "Re-examination of the theory and experimental results of optically induced molecular reorientation and nonlinear diffractions in nematic liquid crystals: spatial frequency and temperature dependence," Phys. Rev., A27, 2747 (1983).

16. I. C. Khoo, G. Finn, R. R. Michael and T. H. Liu, "Passive optical self-limiter using laser induced axially symmetric and asymmetric transverse self-phase modulations in a liquid crystal film," Optics Letters, 11, 227 (1986).

17. I. C. Khoo and R. Normandin, "Nanosecond degenerate optical wave mixing and ultrasonic wave generation in the nematic phase of liquid crystals," Opt. Letts., 9, 285-287 (1984).

18. I. C. Khoo, "Dynamic gratings and the associated self-diffractions and wave front conjugation processes in nematic liquid crystals,” IEEE J. Quant. Electronics, QE22, 1268 (1986).

19. I. C. Khoo, R. R. Michael and G. M. Finn, "Self-phase modulation and optical limiting of a low power CO2 laser with a nematic liquid-crystal film," Appl. Phys. Letts., 52, 2108 (1988).

20. I. C. Khoo, R. G. Lindquist, R. R. Michael, R. J. Mansfield and P. Lopresti, "Dynamics of picosecond laser induced density, temperature and flow-reorientation effects in the mesophases of liquid crystals," J. Appl. Phys., $\underline{69}, 3853$ (1991).

21. Hong Li, Yu Liang and I. C. Khoo, "Transient laser induced orthogonal director axis reorientation in dye-doped liquid crystal,” Mol. Cryst. Liq. Cryst., 251, 85 (1994).

22. I. C. Khoo, Min-Yi Shih, M. V. Wood, B. D. Guenther, and P. H. Chen, F. Simoni, S. Slussarenko*, O. Francescangeli, L. Lucchetti“"Dye-doped photorefractive liquid crystals for dynamic and storage holographic grating formation and spatial light modulation" Invited Paper - IEEE Proceedings Special Issue on Photorefractive Optics: Materials, Devices and Applications. IEEE Proceedings Vol. 87, no. 11, pp $1897-1911$ (1999).

23. I.C. Khoo, S. Slussarenko, B. D. Guenther and W. V. Wood, "Optically Induced Space Charge Fields, DC Voltage , and Extraordinarily Large Nonlinearity in Dye-doped Nematic Liquid Crystals.,” Opt. Letts_23, pp 253 - 255 (1998).

24. I. C. Khoo and J. Ding, "All-optical cw laser polarization conversion at 1.55 micron by two beam coupling in nematic liquid crystal film,” Appl. Phys. Letts. 81, pp. 2496-2498 (2002).

25. I. C. Khoo, "Holographic grating formation in dye- and fullerene C60-doped nematic liquid crystal film", Optics Letters, 20, 2137 (1996)

26. I. C. Khoo, J. Ding, Y. Zhang, K. Chen and A. Diaz, "Supra-Nonlinear Photorefractive Response of Single-wall Carbon Nanotube- and C60-Doped Nematic Liquid Crysta," Appl. Phys. Letts. 82, pp. 3587-3589 (2003).

27. I. C. Khoo, J. Ding, A. Diaz, Y. Zhang and K. Chen, "Recent studies of optical limiting, image processing and near-infrared nonlinear optics with nematic liquid crystals," Mol. Cryst. Liq. Cryst. 375, pp. 33-44 (2002).

28. L.Lucchetti, M.Di Fabrizio, O.Francescangeli, and F. Simoni, "Colossal optical nolinearity in dye-doped liquid crystals," Opt. Comm. 233, 417 (2004).

29. Malgosia Kaczmarek, Andrey Dyaduysha, Sergei Slussarenko and I.C. Khoo, "The role of surface charge field in two-beam coupling in liquid crystal cells with photoconducting polymer layers," J. Appl. Phys. 96, pp. 2616-2623 [2004]

30. E. Graugnard, J. S. King, S. Jain, C. J. Summers, Y. Zhang-Williams and I. C. Khoo, "Electric field tuning of the Bragg peak in large-pore TiO2 inverse shell opals," Phys. Rev. B72, 233105 (2005)

31. I. C. Khoo, Kan Chen and Y. Zhang Williams, "Orientational Photorefractive Effect in undoped and CdSe NanoRods doped Nematic Liquid Crystal - Bulk and Interface Contributions," IEEE J. Selcted Topics in Quantum Electronics JSTQE 12 (3), pp. 443-450 [2006]. 
R. Macdonald, P. Meindl, G. Chilaya and D. Sikharulidze, "Photo-excitation of space charge fields and reorientation of a nematic liquid crystal of discotic molecules," Opt. Comm. 150, 195-200 (1998).

32. P. Pagliusi, R. Macdonald, S. Busch, G. Cipparrone and M. Kreuzer, " Nonlocal dynamic grating and energy transfer by optical two-beam coupling in a nematic liquid crystal owing to highly sensitive photoelectric reorientation," J. Opt. Soc. Am. B18, pp 1632-1638, (2001).

33. I. C. Khoo, M. V. Wood, M. Y. Shih and P. H. Chen, "Extremely Nonlinear Photosensitive Liquid Crystals for Image Sensing and Sensor Protection," Optics Express, Vol. 4, no. 11, pp 431-442 (1999).

34. I. C. Khoo and J. Ding, "All-optical cw laser polarization conversion at 1.55 micron by two beam coupling in nematic liquid crystal film," Appl. Phys. Letts. 81, pp. 2496-2498 (2002); I.C. Khoo and A. Diaz, "Nonlinear dynamics in laser polarization conversion by stimulated scattering in nematic liquid crystal films," Phys. Rev. E68 pp 042701-1 to -4 [2003]

35. I. C. Khoo, M. V. Wood, M. Y. Shih and P. H. Chen, "Extremely Nonlinear Photosensitive Liquid Crystals for Image Sensing and Sensor Protection," Optics Express, Vol. 4, no. 11, pp 431-442 (1999).

36. I. C. Khoo, Jae-Hong Park and Justin Liou, "All-optical Switching of Continuous Wave - Microseconds Lasers with a Dye-doped Nematic Liquid Crystal,” Appl. Phys. Letts. (April issue, 2007). 